\title{
Removal Processing inside PDMS by Short Pulse Laser
}

\author{
Katsuyuki Hayashi ${ }^{* 1}$, Shigeki Matsuo*1 \\ ${ }^{* 1}$ Department of Mechanical Engineering, Shibaura Institute of Technology, 3-7-5 Toyosu, Koto-ku, \\ Tokyo, 135-8548, Japan \\ E-mail: md16069@shibaura-it.ac.jp
}

\begin{abstract}
In this study, we demonstrated removal processing inside polydimethylsiloxane (PDMS) using a short-pulse laser without a photomask. As a light source, a passive Q-switched Nd:YAG laser was used, with a wavelength of $1064 \mathrm{~nm}$ and a pulse width of $500 \mathrm{ps}$. We fabricated micro cavities with a rectangular shape and complex three-dimensional structure. In addition, the fabricated cavity was cut with a cutter knife and the cross section was observed with scanning electron microscopy in order to investigate the processing characteristics in this experimental system. From the observation of the cavity's cross section, we found that the height was smaller than the design value and the upper wall was bent. We considered that thermal expansion during laser processing was the origin. By simultaneously slowing down the stage moving speed and repetition rate, the degree of bending was able to be decreased. Although there was bending of the cross section, we fabricated cavities with a rectangular shape and a complex three-dimensional structure.

DOI: 10.2961/jlmn.2017.02.0016
\end{abstract}

Keywords: PDMS, sub-nanosecond laser, internal processing, 3D processing, microchannel

\section{Introduction}

Polydimethylsiloxane (PDMS) is a silicone-based polymer that is often used to fabricate microfluidic chips. In particular, because of its low-toxicity, PDMS is a suitable material for use in biological microelectromechanical systems (bio-MEMS) where methods for manipulating (mixing, reacting and separating) cells are required [1-3]. In recent years, regenerative medicine has been actively investigating techniques to analyze cells and eggs ranging in size from several tens $\mu \mathrm{m}$ to several $\mu \mathrm{m}$, for example, ES cells and iPS cells [4-5]. Microfluidic chips made of PDMS meet this demand.

As a microprocessing method of PDMS, soft lithography is mainly used. In this method, first, a micropattern of photoresist is fabricated on a substrate such as glass by photolithography using a photomask. The micropatterned photoresist acts as a mold. Next, uncured PDMS is poured into the fabricated mold, and the PDMS is thermally cured. Then, the micro pattern is transferred to the PDMS. Finally, the PDMS with micropatterns engraved onto it is bonded to a glass plate with plasma, and the microfluidic chip is completed [6]. However, in the case of manufacturing a three-dimensional structure using this method, it is necessary to repeatedly carry out the photolithography process using many photomasks for each layer [7]. Alignment of the photomask and the substrate is required for each exposure, and the process is complicated [8]. Because the bonding force between PDMS and the glass substrate is limited, when the pressure inside the microchannel increases, the adhesion peels off and the liquid in the microchannel leaks [9].

In this study, we demonstrated removal processing inside PDMS using short-pulse laser without a photomask. Because the inside of the cured PDMS is directly removed, there is no need to adhere the PDMS to a glass substrate. Consequently, it is more resistant to the internal pressure than the microfluidic chip manufactured by the conventional method. Moreover, the chip has flexibility. We fabricated cavities with a rectangular shape and a complex three-dimensional structure. In addition, the cross section was observed with scanning electron microscopy (SEM), and the processing characteristics in this experimental system were investigated.

\section{Methods and materials}

\subsection{Setup for laser irradiation}

Figure. 1 shows the experimental setup for laser irradiation. As a light source, a passive Q-switched Nd:YAG laser (PNP-M 08010-13, Teem Photonics) was used with a pulse width of 500 ps, a wavelength of $1064 \mathrm{~nm}$, a maximum repetition rate of $1000 \mathrm{~Hz}$, and a pulse energy of $80 \mu \mathrm{J}$. The repetition rate was adjusted by a function generator connected to the laser controller. The pulse energy was adjusted by a half-wave plate (HWP) and a polarizing beam splitter (PBS). The beam diameter was expanded $2 \mathrm{~mm}$ to $10 \mathrm{~mm}$ by a beam expander and introduced to an optical microscope (IX70, Olympus). The laser beam was focused using an objective lens (LCPLN20XIR, NA $=0.45$, Olympus). We calculated the focus diameter using the Airy formula [10]:

$$
d=1.22 \frac{\lambda}{N A},
$$

where $d$ is the focus diameter, $\lambda$ is the wavelength and $N A$ is the numerical aperture of the objective lens. Using Equation 1 and each parameter, the focus diameter was calculated to be about $2.9 \mu \mathrm{m}$.

In addition, the beam waist and Rayleigh length in the Gaussian beam can be expressed by the following equations.

$$
\omega_{0}=\frac{\lambda}{\pi N A},
$$




$$
z=\frac{2 \pi \omega_{0}^{2}}{\lambda}
$$

where $\omega_{0}$ is the beam waist and $z$ is the Rayleigh length. Also, the focal length of the beam can be determined by the following equation:

$$
b=2 z=\frac{4 \pi \omega_{0}^{2}}{\lambda}=\frac{4 \lambda}{\pi N A^{2}},
$$

where $b$ is the focal length of the beam. Through substitution, the focal length of the beam was calculated to be about 6.7 $\mu \mathrm{m}$.

Furthermore, the overlapping ratio indicated by $\eta$ is defined by the following equation:

$$
\eta=\left\{1-\frac{V}{D \cdot F}\right\} \times 100(\%),
$$

where $D$ is the processing diameter, $V$ is the stage moving speed and $F$ is the repetition rate.

The sample on the three-axis motorized stage (XY-axis: ALS-6012-G1M 14J-029R, Z-axis: ALV-600B-HIM 14H008R, Chuo Precision Industrial Co., Ltd) was irradiated with the laser, and the sample was processed by scanning the stage along the pre-designed pattern.

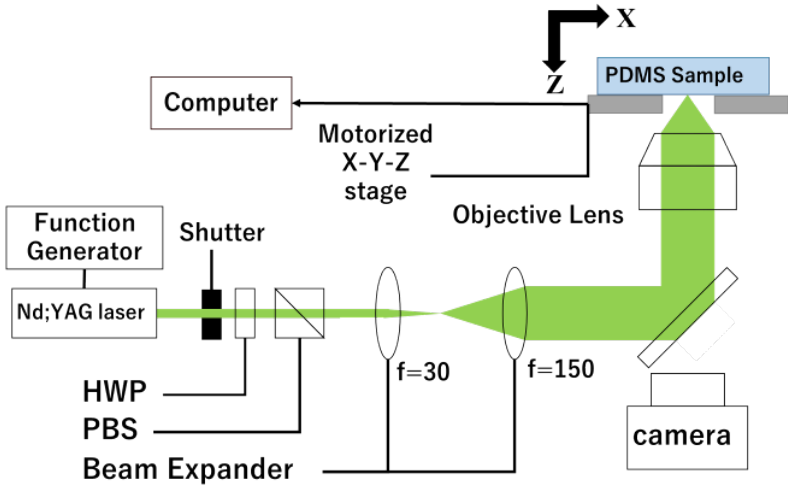

Fig. 1 Setup for laser irradiation.

\subsection{Sample preparation}

The raw materials for the PDMS samples were supplied by DOW CORNING TORAY (SILPOT 184 W/C). Proper amounts of the PDMS base and curing agent were measured in a weight ratio of 10:1 and mixed well. The mixture was put on a dish, and placed in a vacuum chamber to evacuate the bubbles generated during mixing. The degassed PDMS was placed on a hot plate at $90^{\circ} \mathrm{C}$ for 30 minutes to cure. A Transparent sheet was obtained with a thickness of about $7 \mathrm{~mm}$. The cured PDMS was cut with a cutter knife to obtain a square samples sheet of about $3 \mathrm{~cm} \times 3 \mathrm{~cm}$.

\subsection{Measuring method}

During and after laser irradiation, the fabricated patterns were observed with a camera (AIR A01, Olympus) attached to the microscope. In addition, the shape of the cavity processed inside the PDMS was observed with a scanning electron microscope (JSM-6010LV, JEOL) after cutting the PDMS with a cutter knife.

\section{Results and Discussion}

\subsection{Laser-modified lines with single scan}

Before fabrication of the microcavity, we investigated the processing threshold and minimum line width in our experiment setup. First, the PDMS sample was fixed on a motorized three-axis stage, and the laser was focused inside the sample using an objective lens. Second, the stage was moved only once along the Y-axis. The portion irradiated by the laser changed to black, and hereafter it will be referred to as the laser-modified line. Finally, we examined the laser-modified line under the microscope with the camera attached and measured the width.

Figure. 2 shows the results of single line scans, $100 \mu \mathrm{m}$ below the incident surface with different pulse energies and stage moving speeds. In this experiment, the repetition rate was fixed at $1000 \mathrm{~Hz}$. Each line was scanned only once. As shown in Fig. 2, when the pulse energy was $20 \mu \mathrm{J}$ and the stage moving speed was higher than $400 \mu \mathrm{m} / \mathrm{s}$, the processing traces were not continuous. When the pulse energy was higher than $20 \mu \mathrm{J}$, the lines were continuous, while the width became narrower in higher moving speeds. However, when the stage moving speed was high, the vibration during the stage movement became large, which made accurate processing difficult. Figure. 3 plots the measured single ablation line width versus stage moving speed at each irradiation pulse energy. As seen in Fig. 3, the minimum processing width achievable in this experimental setup is about $9.7 \mu \mathrm{m}$ when the pulse energy is $20 \mu \mathrm{J}$ and the stage moving speed is $400 \mu \mathrm{m} / \mathrm{s}$, which corresponds to an overlapping ratio of $96 \%$.

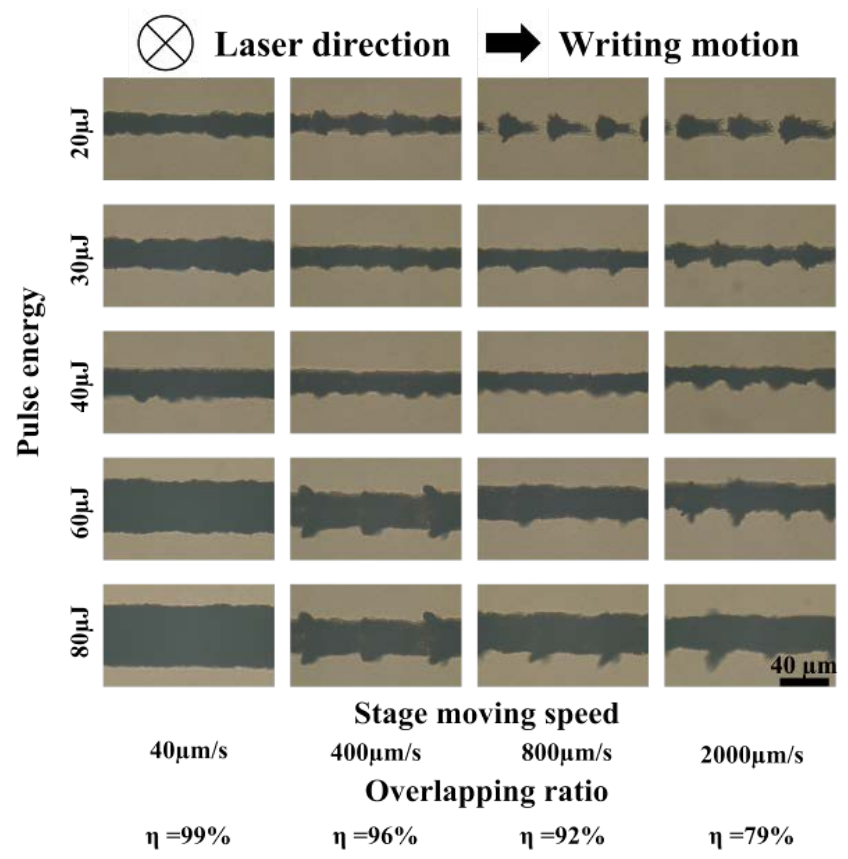

Fig. 2 Microscopic view of laser-modified single lines at the PDMS within $100 \mu \mathrm{m}$ from the surface with different pulse energies and stage moving speeds. The scale bar is $40 \mu \mathrm{m}$, which is common for all. 


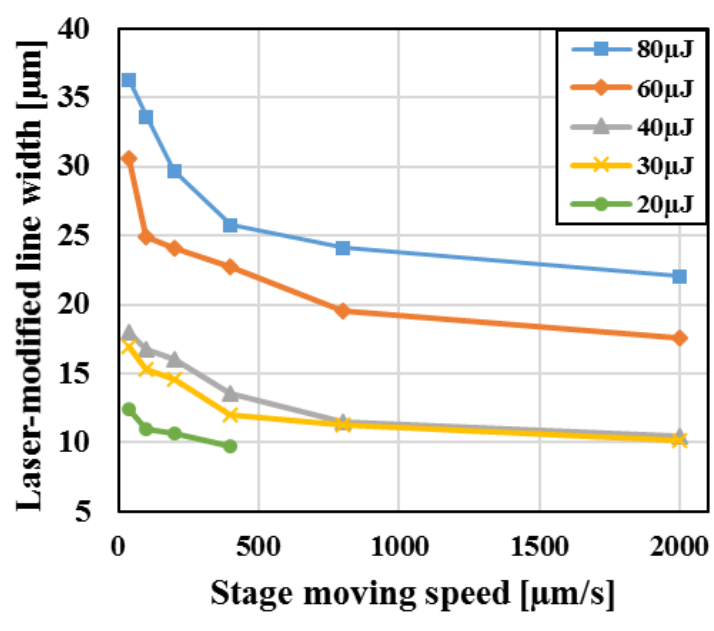

Fig. 3 Plots of the single laser-modified line width versus the stage moving speed.

As seen in Fig. 2, some of the laser modified lines have periodic bursting points, as typically shown when the pulse energy is $20 \mu \mathrm{J}$ and the stage moving speed is $800 \mu \mathrm{m} / \mathrm{s}$ and $2000 \mu \mathrm{m} / \mathrm{s}$. However, the cause of this phenomenon is still unknown. We would like to investigate this phenomenon in the future.

Next, we investigated the focal-depth dependence of the processing characteristics. In this experiment, the repetition rate was $1000 \mathrm{~Hz}$, the pulse energy was $20 \mu \mathrm{J}$, and the stage moving speed was $400 \mu \mathrm{m} / \mathrm{s}$. The focusing position was changed every $100 \mu \mathrm{m}$ from $100 \mu \mathrm{m}$ to $600 \mu \mathrm{m}$ from the incident surface of the PDMS sample, and each line was scanned only once. Figure. 4 shows the microscopic views of the resultant lines. Figure. 5 plots the measured line width versus the focus depth from the incident surface. As shown in Figs. 4 and 5, there was no significant change in the ablation width when the focal position changed. Thus, the virtual dependence of the processing characteristics on the focal position can be eliminated.
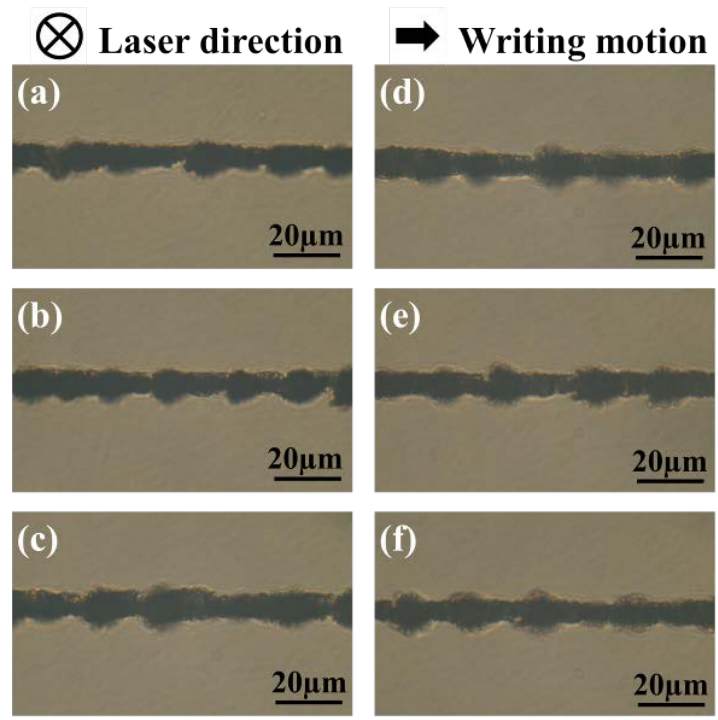

Fig. 4 Microscopic view of laser modified lines at the PDMS within 100-600 $\mu \mathrm{m}$ from the surface: (a) $100 \mu \mathrm{m}$, (b) $200 \mu \mathrm{m}$, (c) $300 \mu \mathrm{m}$, (d) $400 \mu \mathrm{m}$, (e) $500 \mu \mathrm{m}$, and (f) $600 \mu \mathrm{m}$.

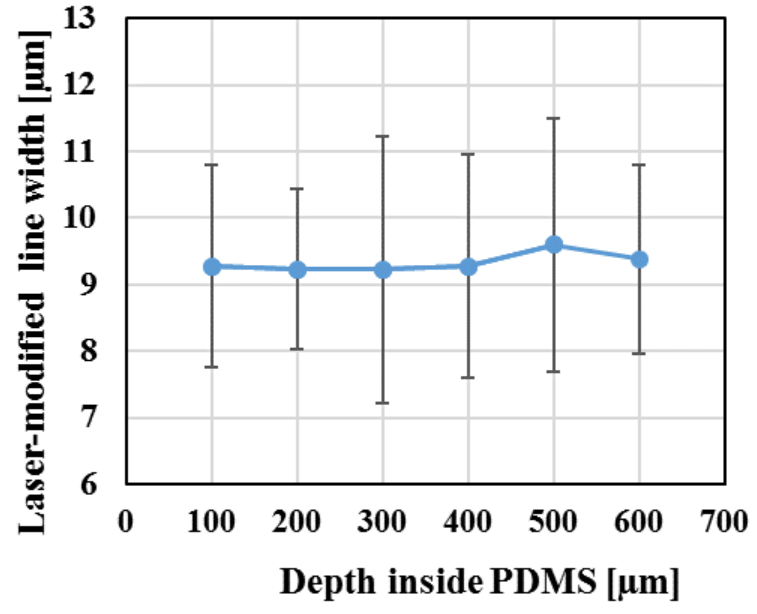

Fig. 5 Plots of the laser modified line width versus the focus position.

\subsection{Cavity processing inside PDMS}

Figure. 6 shows the process of fabricating a cavity inside the PDMS. First, a PDMS sample was fixed on a motorized three-axis stage, and the stage was scanned so that laser irradiation was carried out in the entire removal part of the internal-processing section, which consists of the microcavity. Second, similarly, the stage was scanned so that the laser was irradiated in the region of an inlet and outlet, from inside to surface. Next to the laser irradiation, in order to remove the residue remaining inside the cavity, silicone oil was injected inside the cavity. Then, the sample was immersed in acetone and subjected to an ultrasonic bath $(43 \mathrm{kHz}, 3$ minutes). In order to observe the shape of the processed cavity, the washed PDMS was cut with a cutter knife, and the cross section was observed with a scanning electron microscope. When observing the cross-sectional images (Figs. 79), the PDMS samples were turned upside down so that the inlet and outlet faced upward.

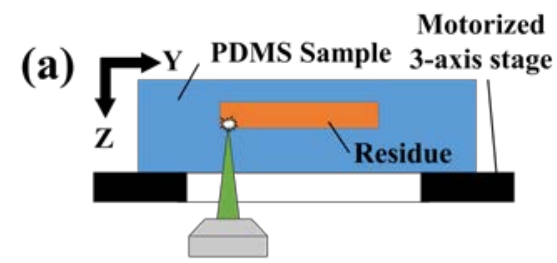

(b)

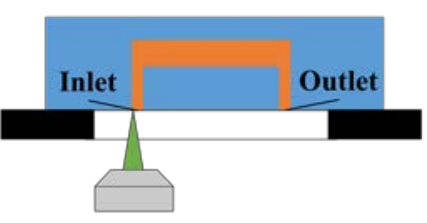

(c)

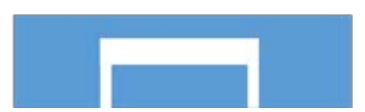

Fig. 6 Process flow to remove laser modified region inside the PDMS: (a) fix PDMS sample on three-axis stage and execute stage control program; (b) process inlet and outlet from the inside to the surface; and (c) remove residue using ultrasonic bath. 
First, we processed a rectangular-shaped microcavity having a width of $400 \mu \mathrm{m}$, a height of $100 \mu \mathrm{m}$ and a length of $800 \mu \mathrm{m}: 800 \mu \mathrm{m}$ lines were drawn in the Y-axis with a 4 $\mu \mathrm{m}$ interval in the $\mathrm{X}$-axis and a $5 \mu \mathrm{m}$ interval in the Z-axis. Next, we processed the inlet and outlet with a width of 50 $\mu \mathrm{m}$, a length of $50 \mu \mathrm{m}$, and a height of $300 \mu \mathrm{m}$ from the inside to the surface from both ends of the cavity with a $4 \mu \mathrm{m}$ interval in $\mathrm{X}$ - and Y-axes. Using an ultrasonic bath treatment, the color of the laser-modified regions changed from black to transparent; this suggested the formation of a microcavity. From the cross-sectional SEM image, we found that microcavities were successfully fabricated.

The cavities had bending in the upper part (the upper part was irradiated at the end), as shown in Fig. 8(a). To our knowledge, such bending has never been observed in rigid materials such as glass. We presumed that expansion due to heat accumulation is responsible for this bending. Figure. 7 shows the scheme of the cavity's bending that we observed. In Fig. 7, the region surrounded by a broken line shows the planned processing region (the region where laser irradiation will be carried out if no thermal expansion occurs). First, the bottom of the planned processing region was irradiated. At this stage, thermal expansion was negligible; thus, the laser was irradiated exactly on the planned region (Fig. 7(a)). At the final stage of the irradiation, the sample thermally expands due to heat accumulation. Then, the laser-irradiated region deviated from the planned processing region (Fig. 7(b)). Finally, after the laser processing was completed, the heat was dissipated, and the sample shrank, resulting in the bending of the upper wall (Fig. 7(c)).

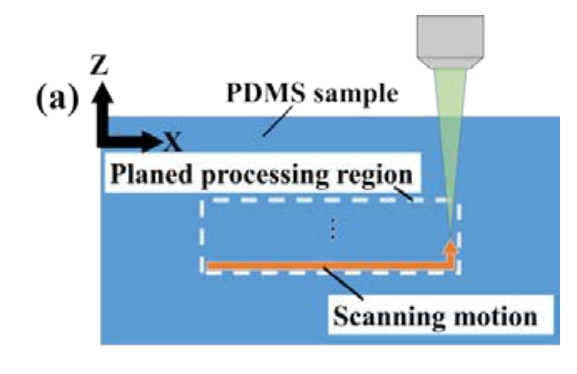

(b)

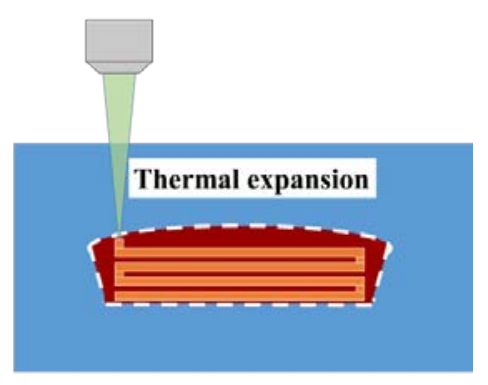

(c)

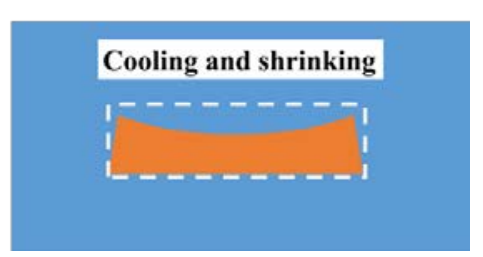

Fig. 7 Scheme of cavity's bending: (a) processing a rectangular cross section; (b) thermal expansion due to rising temperature of neighborhood of laser-focusing point; and (c) sample shrinking and bending.
In order to prove that the bending of the cross section was due to thermal expansion, we processed the same shape with a decreased repetition rate and stage moving speed while keeping the overlapping ratio, and compared the cross-sectional shapes. Figure. 8 shows SEM images of the cross section of the cavity, and Table 1 shows the measured width and height of each cavity's cross section. Fig. 8 and Table 1 show that the width at each stage speed were almost the same. However, the bending of the upper wall was reduced when the stage moving speed slowed. This supports our idea that the bending is due to heat accumulation.
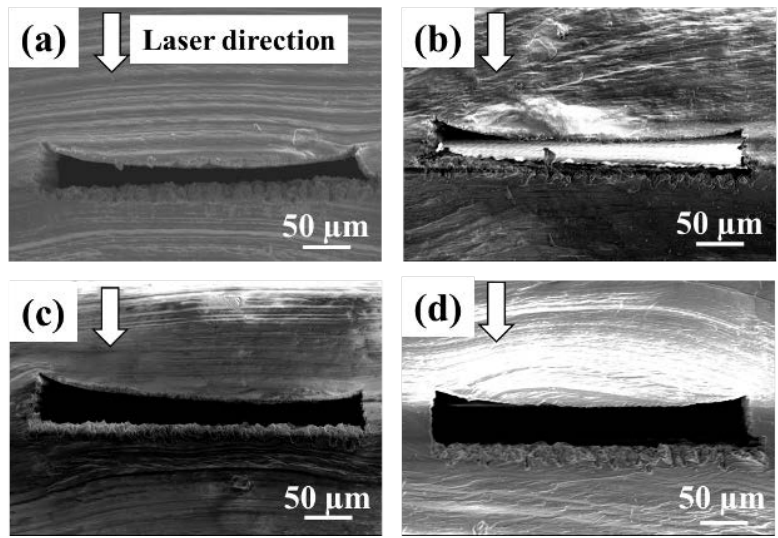

Fig. 8 Relationship between sectional shape of cavity and repetition rate and stage moving speed: (a) $1000 \mathrm{~Hz}$, $400 \mu \mathrm{m} / \mathrm{s}$; (b) $500 \mathrm{~Hz}, 200 \mu \mathrm{m} / \mathrm{s}$; (c) $250 \mathrm{~Hz}$, $100 \mu \mathrm{m} / \mathrm{s}$; and (d) $100 \mathrm{~Hz}, 40 \mu \mathrm{m} / \mathrm{s}$.

Table 1 The width and height of the cross-sectional shape of the cavity at each repetition rate and stage moving speed: (a) $1000 \mathrm{~Hz}, 400 \mu \mathrm{m} / \mathrm{s}$;

(b) $500 \mathrm{~Hz}, 200 \mu \mathrm{m} / \mathrm{s}$; (c) $250 \mathrm{~Hz}, 100 \mu \mathrm{m} / \mathrm{s}$; (d) $100 \mathrm{~Hz}, 40 \mu \mathrm{m} / \mathrm{s}$

\begin{tabular}{|c|c|c|c|c|c|}
\hline & $\begin{array}{c}\text { Design } \\
\text { value }\end{array}$ & (a) & (b) & (c) & (d) \\
\hline Width $[\mu \mathrm{m}]$ & $\mathbf{4 0 0}$ & $\mathbf{3 8 5}$ & $\mathbf{3 8 2}$ & $\mathbf{3 8 7}$ & $\mathbf{3 8 2}$ \\
\hline $\begin{array}{c}\text { Maximum } \\
\text { height }[\mu \mathrm{m}]\end{array}$ & $\mathbf{1 0 0}$ & $\mathbf{6 3}$ & $\mathbf{7 0}$ & $\mathbf{7 4}$ & $\mathbf{9 0}$ \\
\cline { 1 - 5 } $\begin{array}{c}\text { Minimum } \\
\text { height }[\mu \mathrm{m}]\end{array}$ & $\mathbf{3 8}$ & $\mathbf{5 2}$ & $\mathbf{5 3}$ & $\mathbf{6 9}$ \\
\hline
\end{tabular}

\subsection{Fabrication of cavities with complex three-dimen- sional structures}

This method enables manufacturing of a three-dimensional structure which is difficult to process by the photolithography method. Figure. 9 shows an SEM image of the cross section of a three-dimensional structure and its design shape. The design consists of five stacked rectangular cavities with a width of $400 \mu \mathrm{m}$, height of $70 \mu \mathrm{m}$, and length of $800 \mu \mathrm{m}$. With the conventional photolithographic method, this design needs five times the alignment of the photomaskexposure process, but using our method, it needs only the execution of a single-stage control program. As shown in Fig. 
9, although the cross-sectional shape was bent, the complex dimensional microcavity was fabricated.
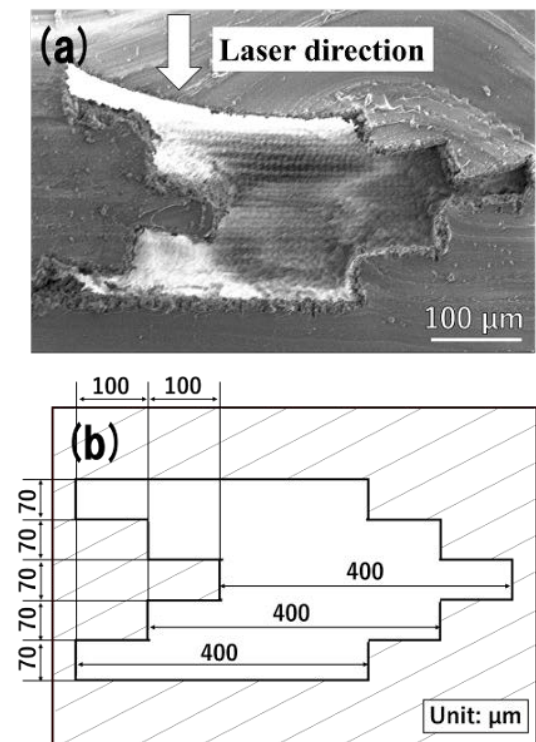

Fig. 9 Cross section of three-dimensional processing: (a)SEM image and (b) design shape.

It is also possible to process multiple channels in one PDMS sample. To demonstrate this, we fabricated overhead-crossing microchannels. First, a zigzag channel with a height of $100 \mu \mathrm{m}$ and width of $150 \mu \mathrm{m}$ was processed at a depth of $600 \mu \mathrm{m}$, and then, a straight channel with a width of $100 \mu \mathrm{m}$ and height of $100 \mu \mathrm{m}$ was processed at $200 \mu \mathrm{m}$ above the top of the zigzag channel. Figure. 10 shows a photograph and design of multiple channels. Red and green liquids in Fig. 10(a) represent, aqueous solutions of rhodamine and uranine dyes, respectively. It was confirmed that the aqueous solutions flowed to the processed channels without contamination.

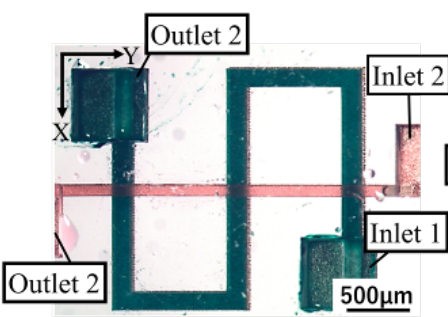

(a)

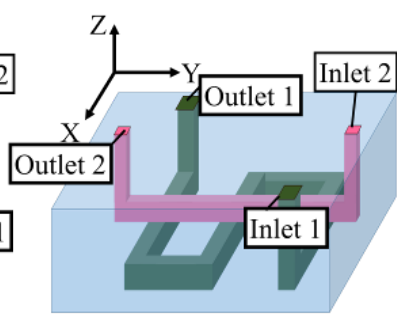

(b)
Fig. 10 The processed multiple channels:

(a) photograph (top view) and (b) 3D design.

\section{Summary}

In the present study, we have succeeded in an internal removal processing of PDMS by using a passive Q-switched Nd:YAG laser. Compared to the conventional photolithography method, this method enables processing of PDMS without using a photomask and photolithography apparatus. In addition, because the processing method does not require a bonding step, even when the internal pressure increases, the bonding does not peel off.

The minimum processing width in a single-line scan was about $9.7 \mu \mathrm{m}$ in this experimental setup. Furthermore, from observation of the cross section of rectangular cavities, we found that the height is smaller than the target value, and the upper wall was bent. We considered that the origin of the bending was thermal influence during laser processing. By simultaneously decreasing the stage moving speed and laser repetition rate, the error of fabrication decreased. We also demonstrated fabrication of a complex three-dimensional structure. The roughness of the cavities was also problem. We plan to improve the processed surface using an etching solution.

\section{References}

[1] A. Manz, N. Graber, and H. M. Widmer, Sens. Actuators B, 1, (1990), 244.

[2] A. R. Prakash, S. Adamia, V. Sieben, P. Pilarski, L.M. Pilarski and C.J. Backhouse, Sens. Actuators B, 113, (2006), 398.

[3] K. J. Regehr, M. Domenech, J. T. Koepsel, K.C. Carver, S. J. Ellison-Zelski, W. L. Murphy, L. A. Schuler, E. T. Alarid and D. J. Beebe, Lab chip, 9, (2009), 2132.

[4] I. Barry, Nature Reviews Cancer, 9, (2009), 230.

[5] H. Kawasaki, K. Mizuseki, S. Nishikawa, S. Kaneko, Y. Kuwana, S. Nakanishi, S. Nishikawa and Y. Sasai Neuron, 28, (2000), 31.

[6] G. M. Whitesides, E. Ostuni, S. Takayama, X. Y. Jiang and D. E. Ingber, Annu. Rev. Biomed. Eng., 3, (2001), 335.

[7] J. R. Anderson, D. T. Chiu, R. J. Jackman, O. Cherniavskaya, J. C. Mcdonald, H. Wu, S. H. Whitesides and G. M. Whitesides, Anal. Chem., 72, (2000), 3158.

[8] H. Becker and C. Gartner, Electrophoresis, 21, (2000), 12.

[9] R. O. Rodrigues, D. Pinho, V. Faustino and R. Lima, Biomed Microdevices, 17, (2015), 108.

[10] J. W. Goodman, Introduction to Fourier Optics, (1996).

(Received: July 22, 2017, Accepted: September 3, 2017) 